\title{
Modelo factorial de inclusión en estudiantes de pedagogía1
}

\section{Factorial model of inclusion among undergraduate teacher-education students}

\author{
ISSN 1510-2432 - ISSN 1688-9304 (en línea) - DOI: 10.18861/cied.2017.8.2.2684
}

Marta Belmar Mellado

Postdoctorado en Sluggish Cognitive Tempo en el ámbito de los trastornos por déficit de atención e hiperactividad en la infancia, Instituto Universitario en Ciencias de la Salud, Universitat de Illes Balears Mallorca, España. Doctora en Diseño, Orientación e Intervención Psicopedagógica, Universidad de Alicante, España. Magister en Ciencias de la Educación, Universidad Católica de la Santísima Concepción, Chile. Licenciada en Educación. Premio Beca Presidente de la República (CONICYT) estudios de Doctorado, 2007. Premio Pasantía Postdoctoral Fundación Carolina, España, 2015. Premio Beca Postdoctoral, Gobierno de Chile, 2017. Profesora, Facultad de Ciencias de la Educación, Universidad Católica del Maule, Chile.

\section{Juan Cornejo Espejo}

Postdoctorado en Historia, Universidad de Santiago de Chile. Postdoctorado en Género y Sexualidad, The Manchester University, Reino Unido. Doctor en Estudios Americanos, Universidad de Santiago de Chile, Chile. Doutor em Ciência Política, Instituto Universitario de Pesquisas de Rio de Janeiro, Brasil. Magíster en Ciencias Sociales, Instituto Latinoamericano de Doctrina y Estudios Sociales - ILADES, Chile. Magíster en Historia, Magíster en Educación, y Profesor de Historia y Geografía Universidad de Santiago de Chile. Magíster en Ciencia Política, Pontificia Universidad Católica de Chile. Licenciado en Ciencias Sociales, Instituto Latinoamericano de Doctrina y Estudios Sociales - ILADES, Chile. Profesor Adjunto, Facultad de Ciencias de la Educación, Universidad Católica del Maule, Chile.

Pilar Contreras Parraguez

Doctora en Didáctica de la Matemática, Universidad de Alicante, España. Magíster en Ciencias de la Educación, Universidad Católica de la Santísima Concepción, Chile. Diploma en Estudios Avanzados, formación en investigación didáctica, área de conocimiento de didáctica de la matemática, Universidad de Alicante, España. Licenciada en Educación, Universidad de Concepción, Chile. Beca Presidente de la República de Doctorado en Universidad de Alicante, 2008. Profesora asistente, Escuela de Educación, Universidad Santo Tomás, Chile. Ex analista de formulación, Sistema de créditos transferibles Chile, área Gestión curricular de postgrado, Universidad Católica del Maule, Chile.

Carlos Mellado Yáñez

Doctor en Psicología y Magister en Psicología Educacional, Pontificia Universidad Católica de Chile. Licenciado en Psicología, Universidad de Chile. Investigador y Profesor asistente, Escuela de Psicología, Universidad Santo Tomás, Chile.

Fecha de recibido: 11/08/2016

Fecha de aceptado: 02/05/2017 


\title{
Resumen
}

La inclusión educativa y la atención a la diversidad estudiantil son actualmente un desafío en los centros educativos puesto que movilizan recursos exigiendo cambios culturales al interior de los mismos. Desde esta perspectiva, la formación de profesores inclusivos es una tarea fundamental en las universidades pues requiere actualizar programas educativos y sensibilizar a la comunidad.

El presente estudio analiza las propiedades psicométricas y la estructura factorial de un instrumento diseñado ad hoc en base a una escala Likert y que mide actitudes hacia la inclusión educativa. Se aplicó la escala a una muestra de 363 estudiantes universitarios de pedagogía chilenos, estableciéndose diferencias según el sexo, la edad y el semestre cursado.

Los resultados revelan que el instrumento presentó un alfa de Cronbach de 0.76 evidenciándose características psicométricas aceptables y una adecuada consistencia interna al tiempo que el AFC permitió correlacionar 5 dimensiones resultantes. Entre los principales hallazgos se encontró que las mujeres tienen actitudes más positivas que los varones en las dimensiones de práctica educativa inclusiva e inclusión en minorías. También se observó que la edad se asocia positivamente con la práctica educativa inclusiva y el derecho a la inclusión al tiempo que el semestre cursado se relacionó positivamente con la práctica educativa inclusiva y la inclusión de minorías, y negativamente con la formación docente inclusiva. Estas evidencias sugieren que si bien los estudiantes poseen actitudes positivas hacia la inclusión educativa, éstas mejoran a medida que progresan en sus estudios adquiriendo mayor experiencia. No obstante, para que dichas actitudes perduren en el tiempo se requeriría del refuerzo social de pares y profesorado y, junto con ello, que los programas educativos de formación de profesores incorporasen aspectos técnicos que permitieran lograr una sociedad más inclusiva acorde a los requerimientos educativos actuales.

Palabras claves: formación profesional, actitud, práctica pedagógica, integración escolar, estudio instrumental.

\begin{abstract}
Educational inclusion and support to student diversity are at present a challenge at schools, since they require resources that demand cultural changes within them. From this perspective, the training of inclusive teachers is a fundamental task that universities have to undertake since it requires updating the educational programs and raise awareness within the community.

This study analyzes the psychometric properties and factor structure of an instrument based on a Likert scale designed for such purpose, measuring the attitudes regarding educational inclusion. The scale was administered to a sample of 363 Chilean university students of teacher training programs, establishing differences according to sex, age, and the semester they were taking at the university.

The results revealed that the instrument presented a Cronbach's alpha 0.76 showing acceptable psychometric properties and adequate internal consistency; while the AFC permitted to correlate 5 resulting dimensions. Among the main findings, women were found to have more positive attitudes than men, in the dimensions of inclusive educational practice and inclusion in minorities. Additionally, age was positively associated with inclusive educational practice and the right to inclusion while the semester that they were taking was positively related to inclusive educational practice and inclusion of minorities; and negatively to inclusive teacher training. These findings suggest that while teacher training students possess positive attitudes towards educational inclusion, they improve as they advance in their education, acquiring more experience. However, for such attitudes to
\end{abstract}


last in time, the social reinforcement of peers and teachers would be required. Jointly, educational teacher training programs should incorporate technical aspects promoting a more inclusive society according to the current educational requirements.

Keywords: professional training, attitude, pedagogical practice, school integration, instrumental study.

\section{Planteamiento del problema}

La educación chilena está viviendo un acelerado proceso de reformas que, entre otros aspectos, implica un mejoramiento de la calidad. Ello supone perfeccionar los procesos educativos y mejorar los aprendizajes de modo de superar las desigualdades y proporcionar más oportunidades a todos los estudiantes, teniendo en cuenta la diversidad. Si bien se señala que "el grupo dominante de una sociedad define los rasgos de la cultura que configuran la comprensión del éxito y del fracaso académico, lo que produce diferencias entre los que aprenden o no" (Arnáiz, 2003, p. 13), se intenta que las prácticas educativas eliminen la indiferencia frente a personas diversas o vulnerables, o bien que se deleguen responsabilidades a otros. Educar en la diversidad "no se basa en la adopción de medidas excepcionales para las personas con necesidades educativas específicas, sino en la adopción de un modelo de currículum que facilite el aprendizaje de todo estudiante en su diversidad" (López Melero, 1995, p. 31). En este sentido Booth (1996) expresa que: “Hay una diversidad dentro de grupos comunes y que ella está vinculada al desarrollo de una educación comunitaria compulsora y universal. Tal perspectiva se preocupa por el incentivo a la participación de todos y con la reducción de todas las presiones excluyentes".

En tal medida la atención a la diversidad da cuenta, por una parte, de motivaciones, intereses, destrezas, estilos de aprendizajes, referentes culturales con sus tradiciones y rituales familiares, conceptos de género y diversidad focalizados en las capacidades, entre otros. Y, por otra parte, considera las exigencias que emergen del mundo cambiante y globalizado como, por ejemplo, nuevas reglas o normativas, conflictos xenofóbicos, fundamentalismo religioso, personas con VIH/SIDA vulnerados entre otros, siendo los mismos un continuo desafío a los derechos humanos (Programa de las Naciones Unidas para el Desarrollo, 2000).

Acoger el contexto escolar en su totalidad implica valorar, incluir y atender a la diversidad favoreciendo la perspectiva inclusiva. Así la educación inclusiva toma fuerza desde un ámbito político pedagógico en un contexto en el que, simultáneamente, se profundizan los procesos de exclusión y se reconocen las dificultades institucionales y pedagógicas para lograr las metas de una educación de calidad (Calvo, 2013). Se entiende la inclusión como una dimensión esencial del derecho a la educación. Y, aun cuando el término surge a partir de una iniciativa vinculada a estudiantes con discapacidad, hoy se extiende ampliamente a todos los estudiantes, definiéndose desde La Organización de las Naciones Unidas para la Educación, la Ciencia y la Cultura (UNESCO) como “Un proceso orientado a responder a la diversidad de los estudiantes incrementando su participación y reduciendo la exclusión en y desde la educación. Está relacionada con la presencia, la participación y los logros de todos los alumnos, con especial énfasis en aquellos que, por diferentes razones, están excluidos o en riesgo de ser marginados" (Blanco, 2008, p. 7).

En este sentido la inclusión como proceso intenta disminuir las barreras que obstaculizan la presencia, la participación y los logros de aprendizaje de todos los estudiantes. Sin embargo, no está exenta de dificultades y paradojas (Blanco 2006; Muñoz, López \& Assaél, 2015). Aun cuando brinda la oportunidad de desarrollar ciertas temáticas y abordar nuevos desafíos que permitan reducir la exclusión social, discriminación y desigualdad educativa tomando como base la educación como un derecho humano y la base de una sociedad más 
justa (Blanco, 2010), para que ello sea posible se deben fomentar prácticas de educación inclusiva, accesibles a todas las personas, y que respondan a la diversidad.

Hay que tener presente que todo el desarrollo cultural hacia la inclusión educativa se relaciona con las creencias de las personas (Muñoz et al., 2015; Pozo et al., 2006), por tanto es necesario comprender las concepciones dominantes que responden a la diversidad e inclusión educativa. Tal conocimiento permitirá "clarificar los cambios necesarios en las creencias y en las culturas para poder promover y desarrollar transformaciones coherentes con una nueva visión política y práctica de la educación" (Muñoz et al., 2015, p. 69). Por tanto, acoger los desafíos de la inclusión educativa implica el dominio de enfoques pedagógicos apropiados y, en el ámbito de la formación docente, supone no solo el desarrollo de conocimientos propios de la disciplina sino también introducir temas tales como necesidades educativas especiales, diversidad, interculturalidad, integración e inclusión, entre otros (Infante, 2010).

En esta misma línea, tal como señalan algunas investigaciones, para promover prácticas inclusivas en la educación es necesario el desarrollo de actitudes y sentimientos positivos hacia el trabajo (Colmenero \& Pegalajar, 2015; Loreman, Forlin \& Sharma, 2007). De acuerdo a la conceptualización de Alcántara (1992) una actitud es el sistema por el cual el ser humano determina su conducta con el medio. En este sentido las investigaciones han reportado que las actitudes de los profesores hacia la diversidad se relacionan también con el éxito en la implementación de políticas inclusivas (Avramidis, Bayliss \& Burden, 2000). Producto del compromiso de los docentes, sus actitudes pueden facilitar o, por el contrario, dificultar la implementación de dichas políticas desentendiéndose y obstaculizando la ejecución de los programas de inclusión. Al respecto, Sales, Moliner \& Sanchiz (2001) señalan que la actitud de la comunidad escolar, y en especial las expectativas de los profesores, se relacionan poderosamente con el desarrollo de modelos inclusivos en las escuelas. De hecho, los estudios en el área sugieren que actitudes negativas de los profesores hacia la inclusión generan bajas expectativas en relación a una persona con discapacidad, lo que a su vez reduce las oportunidades de aprendizaje de dicha persona, traduciéndose finalmente en un bajo desempeño (Campbell, Gilmore \& Cuskelly, 2003).

Aun cuando existe consenso internacional sobre lo que implica la educación inclusiva, la realidad muestra que la presencia de múltiples perspectivas termina restándole fuerza (Echeita, 2013), ya sea por desinformación o bien porque se tiende a pensar en inclusión como sinónimo de necesidades educativas especiales, desconociéndose los avances en la perspectiva y en los sustentos teóricos que la investigación ha aportado. En esa línea, un área que ha atraído la atención de los investigadores es el estudio de las actitudes de los estudiantes de pedagogía hacia la inclusión (Campbell et al., 2003). Bajo esta mirada Sales, Moliner \& Sanchiz (2001) afirman que la formación inicial de los profesores debe fomentar actitudes dirigidas a la atención y aceptación de la diversidad para integrar a los alumnos con necesidades educativas especiales. Igualmente Sanya et al. (2005) reportan que las actitudes de los profesores hacia la diversidad se relacionan con su formación inicial, ya que experiencias formativas que sensibilicen sobre las necesidades educativas especiales permiten conformar una comunidad educativa bien informada y tolerante.

Otra perspectiva señala que la mayoría de las investigaciones abordan la percepción de los docentes y del personal administrativo respecto a la inclusión (Zambrano, Córdoba y Arboleda, 2012) indicando que es necesario ampliar este conocimiento ya que los estudiantes son actores fundamentales en el proceso de la inclusión educativa. De este modo es importante conocer las actitudes de los estudiantes de pedagogía acerca de la inclusión educativa para poder desarrollar en ellos un positivo sentido de la inclusión 
tempranamente en el desarrollo personal (Tait \& Purdie, 2000).

Si bien diversas investigaciones han desarrollado instrumentos para medir la percepción hacia la inclusión, la mayoría se centra en las actitudes que muestran los profesores a través de cuestionarios construidos ad-hoc. Por ejemplo, Colmenero y Pegalajar (2015) revisan seis cuestionarios acerca de actitudes hacia la inclusión y todos se encuentran dirigidos a profesores. Por su parte, Zambrano et al. (2012) detectan que la mayoría de los cuestionarios están enfocados a las actitudes de los profesores en ejercicio, así como también dan cuenta de algunos cuestionarios diseñados específicamente para evaluar las actitudes hacia la inclusión en estudiantes de maestría (Colmenero y Pegalajar, 2015), en estudiantes de secundaria (Zambrano et al., 2012) o bien evalúan aspectos específicos de la inclusión como el síndrome de Down (Campbell et al., 2003).

De tal manera el presente trabajo pretende construir y validar un cuestionario desarrollado ad hoc para analizar las actitudes y percepciones sobre la inclusión en estudiantes de pedagogía de la Universidad Católica del Maule (Chile) y si las mismas se ven afectadas por las variables sexo, edad o nivel educativo. Ello en vista de implementar políticas que posibiliten un desarrollo profesional armónico, además de mejorar las condiciones laborales así como la revisión de los sistemas de formación y las claves de la profesión". (Vezub, 2007).

\section{Metodología \\ Participantes}

El estudio de tipo instrumental (Carretero-Dios y Pérez, 2005) describe el proceso de construcción y evaluación de las propiedades psicométricas de un cuestionario desarrollado ad hoc para analizar las actitudes hacia la inclusión de estudiantes de pedagogía. La investigación se llevó a cabo en la Universidad Católica del Maule en Chile (UCM) con estudiantes de ocho carreras de pedagogía a través un muestreo no probabilístico por conveniencia, el cual incluye en la muestra a los participantes seleccionados por su disponibilidad (Cardona, 2002). Se obtuvo información de 363 alumnos (70,5\% mujeres, $29,5 \%$ varones) cuya media de edad fue de 21,52 (D.S = 2,57) para los varones y de 21,10 $(D . S .=3,18)$ para las mujeres. Las carreras con mayor representación fueron Pedagogía en Educación Física (19,3\%), Pedagogía en Matemática (16,5\%), Pedagogía en Castellano (15,4\%), Educación de Párvulos (14,3\%) y Pedagogía Básica (13,2\%), mientras que las carreras con menor representación fueron Pedagogía en Religión y Filosofía (2,2\%) y Pedagogía en Ciencias (7,7\%). La mayoría de los alumnos de la muestra cursaban primero, segundo y tercer año $(92,8 \%)$ de sus respectivas carreras.

\section{Procedimiento}

La aplicación del cuestionario a los estudiantes de la UCM se realizó en el aula de clase, de manera voluntaria, individual y anónima, con la presencia de uno de los investigadores en caso de que los alumnos tuvieran alguna duda con respecto a las preguntas. El procedimiento de recolección de los datos respetó las normas éticas de la Declaración de Helsinki, principalmente en lo relativo al consentimiento informado, a la confidencialidad en el uso de los datos y a la voluntariedad en la participación.

\section{Instrumentos}

Para la recolección de la información se confeccionó una escala de actitudes hacia la inclusión compuesta por 30 reactivos a los cuales se respondía expresando el grado de acuerdo utilizando una escala tipo Likert (donde 1 indicaba total desacuerdo y 5 muy 
de acuerdo). Se diseñó una escala ad hoc sustentada en conceptos teóricos atingentes a la inclusión y exclusión educativa, puesto que instrumentos similares utilizados en investigaciones previas fueron diseñados específicamente para profesores que están ejerciendo su práctica pedagógica y no para estudiantes de pedagogía.

El procedimiento de construcción del instrumento consideró dos etapas. En una primera etapa se adaptaron dimensiones e ítems de instrumentos similares utilizados para medir inclusión en profesores (Cardona, 2006; Sanhueza, Granada y Bravo, 2012). La adaptación básicamente consistió en seleccionar las dimensiones y los ítems más idóneos para el contexto universitario y adecuarlos al vocabulario propio del grupo etario al cual pertenece la muestra (i.e. alumnos universitarios). En una segunda etapa se realizó un focus group con estudiantes de pedagogía de la UCM, y en base a los resultados del mismo se definieron nuevas dimensiones (prácticas docentes, derechos en el contexto educativo, exclusión educativa, formación docente y exclusión social) y se redactaron sus correspondientes ítems.

Del problema de investigación, y tal como sostienen Carretero-Dios y Pérez (2005), se desprenden las siguientes variables, las cuales pueden agruparse en seis tipos: 1) variables demográficas, 2) variables relativas a la práctica docente, 3) variables relativas a la formación docente, 4) variables relativas a la inclusión de minorías, 5) variables relativas a la exclusión sociocultural, y 6) variables relativas a los derechos en el contexto educativo.

La delimitación conceptual de éstas se basa en "una afirmación genérica de lo que el constructo es y que a su vez se basa en otros constructos igualmente no delimitados" (Carretero-Dios y Pérez, 2007. p. 869).

1) Variables demográficas. Estas variables recogen información relativa al sexo, edad, carrera y nivel educativo.

2) Variables relativas a la práctica docente. Estas variables recogen información relativa a las actitudes y conceptualización de inclusión. Algunos ejemplos son:

- Ítem 13. La inclusión educativa exige un cambio de actitud de parte de los profesores. - Ítem 17. Un currículo o plan de estudios rígido obstaculiza la inclusión educativa.

3) Variables relativas a la formación docente. Recogen información relativa a la formación docente para atender a la diversidad educativa, así como las implicancias de la inclusión en la labor del profesor. Dos ejemplos son:

- Ítem 10. La UCM prepara a sus futuros profesores para lidiar con estudiantes que son objeto de discriminación y/o exclusión en los sistemas escolares formales.

- Ítem 3. Los profesores de Chile tienen las competencias para trabajar con estudiantes con capacidades distintas.

4) Variables relativas a la inclusión de minorías. Recogen información alusiva a la diversidad en el contexto educativo. Dos ejemplos de ellos son:

- Ítem 14. La inclusión educativa es viable en sectores rurales o socialmente vulnerables. - Ítem 12. Las escuelas deberían aceptar estudiantes transexuales para educar en la diversidad.

5) Variables relativas a exclusión sociocultural. Recogen información relativa a la aceptación de las diferentes culturas e integración en el sistema educativo. Dos ejemplos son:

- Ítem 7. Los estudiantes pertenecientes a grupos étnicos minoritarios o a pueblos originarios no deberían ser asimilados a la cultura nacional. 
- Ítem 8. La inclusión educativa no es una forma de integración al sistema escolar.

6) Variables relativas a los derechos en el contexto educativo. Recogen información concerniente al derecho a la educación y sus bases filosóficas. Ejemplos de ello son:

- Ítem 2. La inclusión educativa se fundamenta en los derechos humanos y en el derecho a la educación.

- Ítem 1. La atención a las necesidades educativas especiales se considera una forma de inclusión educativa.

Si bien los 30 ítems construidos inicialmente permitieron realizar un análisis factorial adecuado, se optó por seleccionar aquellos ítems que presentaron mejor bondad de ajuste y correlación interfactorial, seleccionándose 19 ítems categorizados dentro de: práctica educativa inclusiva, derecho a la inclusión, inclusión de minorías, formación docente inclusiva y exclusión sociocultural. La confiabilidad medida a partir de la distribución de los 19 ítems en esta investigación permitió validar los constructos teóricos del instrumento resultando un alfa de Cronbach general de 0.76, el cual se considera adecuado (Frías, 2011; Huh, Delorme \& Reid, 2006).

\section{Plan de análisis}

En una primera etapa se realizaron análisis descriptivos básicos para caracterizar a los ítems que componen cada subescala. En una segunda etapa se evaluaron las propiedades psicométricas del instrumento. Para evaluar la validez de constructo se utilizó el Análisis Factorial Confirmatorio (AFC). El AFC permite testear hipótesis acerca de la relación entre variables observadas (i.e. ítems) y factores o variables latentes. Por lo mismo, es una técnica adecuada para desarrollar instrumentos de medida y evaluar su validez de constructo (Jackson, Gillaspy \& Purc-Stephenson, 2009). El AFC empieza con la definición de un constructo y con la selección de indicadores. Así, es necesario especificar el número de factores en el modelo y la correspondencia de cada indicador con uno de los factores determinados teóricamente, sin permitir que exista correlación entre los errores (Kline, 2013). El AFC presenta ciertas ventajas frente al Análisis Factorial Exploratorio (AFE) cuando se trata de establecer la dimensionalidad de un constructo. En primer lugar presenta mayor flexibilidad para especificar el número de factores y establecer los patrones de relaciones entre los ítems y los factores, estableciendo el error asociado. En segundo lugar, la aceptabilidad del modelo especificado puede ser testeada a través de indicadores de bondad de ajuste (Brown, 2006). Para medir la confiabilidad se utilizó el análisis de consistencia interna (alfa de Cronbach). Posteriormente se realizó un análisis de comparación de medias para establecer diferencias entre varones y mujeres y un análisis de correlación y de regresión para establecer la relación entre la edad, el sexo, el semestre cursado y las cinco dimensiones de inclusión. En cada uno de los análisis inferenciales se utilizaron pruebas de significancia estadística. La significancia adoptada fue de p<0,05. Finalmente se sistematizó la información recolectada y se realizó el análisis estadístico con el programa Spss versión 20 y el Programa Mplus 6.11.

\section{Resultados}

En un primer momento se realizaron análisis estadísticos descriptivos básicos de los ítems que componen el instrumento. En la tabla 1 se reportan los estadísticos descriptivos para cada uno de los ítems de la dimensión práctica educativa inclusiva. En general se observa que un alto porcentaje de alumnos se mostraron indiferentes con respecto a los ítems que componen esta dimensión. Las excepciones son los ítems 15 y 17 en los que los alumnos indicaron mostrarse, en su mayoría, de acuerdo y totalmente de acuerdo. 
Tabla 1. Reactivos de la dimensión práctica educativa inclusiva (Descriptivos)

\begin{tabular}{cccccccc}
\hline & & & CD & ED & I & DA & TA \\
\hline ÍTEMS & $\boldsymbol{M}$ & DS & $\%$ & $\%$ & $\%$ & $\%$ & $\%$ \\
13 & 3,26 &, 64 & 0,3 & 0,6 & 82,4 & 6,9 & 9,9 \\
15 & 3,87 &, 80 & 0,0 & 0,3 & 38,6 & 35,0 & 26,2 \\
16 & 3,55 &, 72 & 0,0 & 1,1 & 55,4 & 30,9 & 12,7 \\
17 & 4,03 &, 82 & 0,0 & 0,0 & 32,2 & 32,5 & 35,3 \\
18 & 3,17 &, 49 & 0,0 & 0,8 & 85,7 & 9,4 & 4,1 \\
19 & 3,30 &, 57 & 0,0 & 1,4 & 71,9 & 22,0 & 4,7 \\
\hline
\end{tabular}

Nota. $\mathrm{M}=$ media, $\mathrm{DS}=$ desviación estándar, $\mathrm{CD}=$ completamente en desacuerdo, $\mathrm{ED}=$ en desacuerdo, I = indiferente, $\mathrm{DA}=$ de acuerdo, TA = totalmente de acuerdo.

Para la subescala total $M=3,52 ;$ D.S. $=, 34$

En la tabla 2 se reportan los estadísticos descriptivos para cada uno de los ítems de la dimensión derecho a la inclusión. Nuevamente se observa que un alto porcentaje de alumnos se mostraron indiferentes con respecto a los ítems que componen esta dimensión. La excepción es el ítem 1 en el que los alumnos indicaron mostrarse, en su mayoría, de acuerdo y totalmente de acuerdo.

Tabla 2. Reactivos de la dimensión derecho a la inclusión (Descriptivos)

\begin{tabular}{|c|c|c|c|c|c|c|c|}
\hline & & & $C D$ & ED & I & $\mathrm{DA}$ & TA \\
\hline ÍTEMS & $M$ & DS & $\%$ & $\%$ & $\%$ & $\%$ & $\%$ \\
\hline 1 & 4,32 & ,71 & 0,0 & 0,0 & 14,9 & 38,6 & 46,6 \\
\hline 2 & 3,60 & ,76 & 0,0 & 0,0 & 56,7 & 26,4 & 16,8 \\
\hline 6 & 3,26 & ,58 & 0,0 & 0,3 & 80,2 & 12,4 & 7,2 \\
\hline
\end{tabular}

Nota. $\mathrm{M}=$ media, $\mathrm{DS}=$ desviación estándar, $\mathrm{CD}=$ completamente en desacuerdo, $\mathrm{ED}=\mathrm{en}$ desacuerdo, I = indiferente, DA = de acuerdo, TA = totalmente de acuerdo.

Para la subescala total $M=3,72 ;$ D.S. $=, 44$

En la tabla 3 se reportan los estadísticos descriptivos para los ítems de la dimensión inclusión de minorías. Se observa una tendencia favorable de los alumnos a los ítems correspondientes a esta dimensión, con un alto porcentaje de los participantes mostrándose de acuerdo y totalmente de acuerdo. La excepción es el ítem 14 para el que los alumnos, en su mayoría, se mostraron indiferentes.

Tabla 3. Reactivos de la dimensión inclusión de minorías (Descriptivos)

\begin{tabular}{cccccccc}
\hline & & & CD & ED & I & DA & TA \\
\hline ÍTEMS & $\boldsymbol{M}$ & $\mathbf{D S}$ & $\%$ & $\%$ & $\%$ & $\%$ & $\%$ \\
4 & 4,15 &, 76 & 0,0 & 0,0 & 23,1 & 39,1 & 37,7 \\
5 & 4,48 &, 71 & 0,0 & 0,3 & 12,4 & 26,7 & 60,6 \\
9 & 4,18 &, 81 & 0,0 & 0,0 & 25,6 & 31,1 & 43,3 \\
12 & 4,77 &, 48 & 0,0 & 0,0 & 3,0 & 16,8 & 80,2 \\
14 & 3,33 &, 68 & 0,0 & 1,4 & 74,7 & 13,2 & 10,7 \\
\hline
\end{tabular}

Nota. $\mathrm{M}=$ media, $\mathrm{DS}$ = desviación estándar, $\mathrm{CD}=$ completamente en desacuerdo, $\mathrm{ED}=$ en desacuerdo, $\mathrm{I}$ = indiferente, $\mathrm{DA}=$ de acuerdo, TA = totalmente de acuerdo.

Para la subescala total $M=4,18 ; D . S .=, 37$ 
En la tabla 4 se observan los estadísticos descriptivos para los ítems de la dimensión formación docente inclusiva. Nuevamente se observa una tendencia favorable de los alumnos hacia los ítems correspondientes a esta dimensión, en los que la mayoría de los participantes indica mostrarse de acuerdo y totalmente de acuerdo.

Tabla 4. Reactivos de la dimensión formación docente inclusiva (Descriptivos)

\begin{tabular}{|c|c|c|c|c|c|c|c|}
\hline & & & $C D$ & ED & I & DA & TA \\
\hline ÍTEMS & $M$ & DS & $\%$ & $\%$ & $\%$ & $\%$ & $\%$ \\
\hline 3 & 4,4 & 68 & 0,0 & 0,0 & 19,6 & 47,4 & 33,1 \\
\hline 10 & 4,13 & 71 & 0,0 & 0,0 & 11,6 & 36,9 & 51,5 \\
\hline
\end{tabular}

Nota. $M=$ media, $\mathrm{DS}=$ desviación estándar, $\mathrm{CD}=$ completamente en desacuerdo, $\mathrm{ED}=$ en desacuerdo, I = indiferente, DA = de acuerdo, TA = totalmente de acuerdo.

Para la subescala total $M=4,26 ;$ D.S. $=, 54$

En la tabla 5 se reportan los estadísticos descriptivos para cada uno de los ítems de la dimensión exclusión socio cultural. Se observa una tendencia favorable de los alumnos hacia los ítems correspondientes a esta dimensión, con un alto porcentaje de los participantes mostrándose de acuerdo y totalmente de acuerdo.

Tabla 5. Reactivos de la dimensión exclusión socio cultural (Descriptivos)

\begin{tabular}{|c|c|c|c|c|c|c|c|}
\hline & & & $C D$ & ED & I & DA & TA \\
\hline ÍTEMS & $M$ & DS & $\%$ & $\%$ & $\%$ & $\%$ & $\%$ \\
\hline 7 & 4,50 & ,68 & 0,0 & 0,0 & 11,0 & 28,4 & 60,6 \\
\hline 8 & 4,08 & 81 & 0,0 & 0,3 & 25,3 & 42,1 & 31,7 \\
\hline 11 & 4,30 & ,81 & 0,0 & 0,6 & 20,7 & 27,0 & 51,8 \\
\hline
\end{tabular}

Nota. $\mathrm{M}=$ media, $\mathrm{DS}$ = desviación estándar, $\mathrm{CD}=$ completamente en desacuerdo, $\mathrm{ED}=$ en desacuerdo, I = indiferente, DA = de acuerdo, TA = totalmente de acuerdo.

Para la subescala total $M=4,29 ;$ D.S. $=, 514$

En una segunda etapa se realizó un AFC para evaluar la validez de constructo y la dimensionalidad del instrumento de inclusión. De esta forma se evaluó el ajuste de la estructura dimensional propuesta de cinco factores, con los datos empíricos obtenidos, a través del método de mínimos cuadrados ponderados robustos (WLSMV, Weighted Least Squares Mean and Variance Adjusted). En general se recomienda utilizar más de un índice de bondad de ajuste para evaluar el modelo propuesto. Por ello se utilizaron los siguientes: el estadístico chi-cuadrado, en que valores no significativos indican un buen ajuste de los datos; el Tucker-Lewis Index (TLI) y el Comparative Fit Index (CFI), en que valores superiores a 0,9 indican un buen ajuste entre los datos y el modelo; y finalmente el Root Mean Standard Error of Approximation (RMSEA), en que valores cercanos a 0,05 indican un error aceptable y por lo mismo un muy buen ajuste entre datos y modelo. Los valores de la bondad de ajuste del modelo propuesto fueron: X2 (171) = 2575,45 p < 0,001; RMSEA=0,057; $\mathrm{TLI}=0,91$ y $\mathrm{CFI}=0,93$. Si bien el estadístico chi-cuadrado fue significativo -lo que indica un ajuste no óptimo del modelo- dicha significación puede deberse al tamaño de la muestra. Por lo demás, los valores de los restantes índices permiten concluir que hay un buen ajuste entre el modelo propuesto y los datos observados. 
La Tabla 6 muestra las cargas factoriales estandarizadas de los ítems correspondientes a los cinco factores de la escala de inclusión. Para los cinco factores las cargas de los ítems son significativas $(p<0,001)$ y en general se observan balanceadas y confiables.

Tabla 6. Matriz de cargas factoriales estandarizadas

\begin{tabular}{cccccc}
\hline Ítems & F1 & F2 & F3 & F4 & F5 \\
\hline 13. &, 68 & & & & \\
15. &, 76 & & & & \\
16. &, 65 & & & & \\
17. &, 72 & & & & \\
18. &, 82 & & & & \\
19. &, 84 & & & & \\
1. & &, 49 & & & \\
2. & &, 46 & & & \\
6. & &, 63 & & & \\
4. & & &, 65 & & \\
5. & & &, 62 & & \\
9. & & &, 66 & & \\
12. & & &, 51 & & \\
14. & & &, 62 & & \\
3. & & & &, 69 & \\
10. & & & &, 61 & \\
7. & & & & &, 83 \\
8. & & & & &, 50 \\
11. & & & & &, 76 \\
\hline
\end{tabular}

Nota. F=Factor

La Tabla 7 muestra las correlaciones entre los cinco factores que dan cuenta de la varianza de los ítems del instrumento de inclusión. Se puede observar que la dimensión F1 se correlaciona de forma positiva con las dimensiones F2 y F3 y de forma negativa con la dimensión F4. La dimensión F2, en tanto, se correlaciona de forma positiva con las dimensiones F3 y F5. La dimensión F3 se relaciona de forma positiva con la dimensión F5 y finalmente la dimensión F4 se relaciona positivamente con la dimensión F5.

Tabla 7. Matriz de correlaciones

\begin{tabular}{l|c|c|cc|cc|}
\hline & 1 & 2 & 3 & $\mathbf{4}$ & $\mathbf{5}$ \\
\hline F1 Práctica educativa inclusiva & 1 & & & \\
\hline F2 Derecho a la inclusión &, $57 * *$ & 1 & & & \\
\hline F3 Inclusión de minorías &, $64 * *$ &, $57 * *$ & 1 & & \\
\hline F4 Formación docente inclusiva &,$- 22^{* *}$ &, 20 &, 08 & 1 & \\
F5 Exclusión sociocultural &, 02 &, $31 * *$ &, $32 * *$ &, $57^{* *}$ & 1 \\
\hline
\end{tabular}

Nota. Las correlaciones están aproximadas al segundo decimal. $* * p<.01$. 
En una tercera etapa se evaluó bivariadamente la relación entre las variables sociodemográficas y las dimensiones de inclusión, mediante un análisis t de Student para diferencias de medias en el caso de la variable categórica (sexo) y un análisis de correlación en el caso de las variables continuas (edad y semestre cursado).

En la tabla 8 se presentan los resultados de las correlaciones entre la edad y el semestre cursado por el estudiante y las dimensiones de la inclusión y las diferencias de medias según sexo en dichas dimensiones. Se observó que la edad se relaciona positivamente con la práctica educativa inclusiva y el derecho a la inclusión. El semestre cursado por el estudiante, en tanto, se relacionó positivamente con la práctica educativa inclusiva y la inclusión de minorías, y negativamente con la formación docente inclusiva. Además se observaron diferencias por sexo en las dimensiones práctica educativa inclusiva e inclusión en minorías. Las puntuaciones medias obtenidas por las mujeres en estas dos áreas fueron mayores.

Tabla 8. Matriz de correlaciones entre variables de interés y diferencias de media entre varones y mujeres.

\begin{tabular}{|c|c|c|c|c|c|c|c|c|c|c|c|c|c|c|c|}
\hline & \multicolumn{3}{|c|}{$\begin{array}{l}\text { Práctica } \\
\text { educativa } \\
\text { inclusiva }\end{array}$} & \multicolumn{3}{|c|}{$\begin{array}{l}\text { Derecho } \\
\text { a la } \\
\text { inclusión }\end{array}$} & \multicolumn{3}{|c|}{$\begin{array}{l}\text { Inclusión } \\
\text { de } \\
\text { minorías }\end{array}$} & \multicolumn{3}{|c|}{$\begin{array}{l}\text { Formación } \\
\text { docente } \\
\text { inclusiva }\end{array}$} & \multicolumn{3}{|c|}{$\begin{array}{l}\text { Exclusión } \\
\text { socio } \\
\text { cultural }\end{array}$} \\
\hline & b & ES & $\mathbf{R}^{2}$ & b & ES & $\mathbf{R}^{2}$ & b & ES & $\mathrm{R}^{2}$ & b & ES & $\mathbf{R}^{2}$ & b & ES & $\mathbf{R}^{2}$ \\
\hline Paso 1 & & &, $124 * * *$ & & & ,015 & & &, $049 * * *$ & & &, $029 * \star *$ & & & ,002 \\
\hline Edad &, 013 &, 009 & & ,017 &, 009 & &, 012 &, 010 & &,- 004 &, 010 & &, 005 & ,008 & \\
\hline Sexo &, $250 * \star \star$ & ,053 & &,- 057 & ,058 & &, $187^{* *}$ &, 059 & &, 014 & ,059 & & ,029 & ,051 & \\
\hline Sem. &, $099 * \star *$ &, 023 & & ,006 & ,025 & &, $053^{*}$ &, 025 & &,- 074 ** &, 026 & &,- 010 & ,022 & \\
\hline
\end{tabular}

\begin{tabular}{|c|c|c|c|c|c|c|c|c|c|c|c|}
\hline & 1 & 2 & 3 & 4 & 5 & 6 & 7 & $\begin{array}{l}M(D E) \\
\text { Mujeres } \\
(n=252)\end{array}$ & $\begin{array}{l}M(D E) \\
\text { Varones } \\
(n=111)\end{array}$ & $\begin{array}{l}t \\
(g \mid=361)\end{array}$ & $p$ \\
\hline Edad & -- & & & & & & & $\begin{array}{l}21,08 \\
(3.21)\end{array}$ & $\begin{array}{l}21,59 \\
(2,54)\end{array}$ & $-1,50$ & 133 \\
\hline Semestre &, $37 * *$ & -- & & & & & & $\begin{array}{l}2,03 \\
(1,09)\end{array}$ & $\begin{array}{l}2,04 \\
(1,25)\end{array}$ &,- 06 & 949 \\
\hline $\begin{array}{l}\text { P. educativa } \\
\text { inclusiva }\end{array}$ &, $14^{\star \star}$ &, $25^{\star \star}$ & -- & & & & & $4,53(, 45)$ & $4,29(, 53)$ & 4,42 ** & $<, 001$ \\
\hline $\begin{array}{l}\text { Derecho a } \\
\text { inclusión }\end{array}$ &, $11 *$ & ,05 &, $030 * \star$ & -- & & & & $4,31(, 50)$ & $4,38(, 51)$ & $-1,13$ & ,258 \\
\hline $\begin{array}{l}\text { Inclusión } \\
\text { minorías }\end{array}$ & ,09 &, $14 * \star$ &, $45 * *$ &, $30 * *$ & -- & & & $4,05(, 54)$ & $3,87(, 46)$ & $3,06 * \star$ & ,002 \\
\hline $\begin{array}{l}\text { F. docente } \\
\text { inclusiva }\end{array}$ &,- 08 &,$- 16^{\star \star}$ &,$- 13 * \star$ & ,07 & ,03 & -- & & $3,41(, 55)$ & $3,39(, 45)$ & ,27 & ,785 \\
\hline $\begin{array}{l}\text { Exclusión } \\
\text { socio } \\
\text { cultural }\end{array}$ & ,02 &,- 01 & ,02 &, $14^{* \star}$ &, $15^{\star \star}$ &, $27 * \star$ & -- & $3,26(, 45)$ & $3,23(, 42)$ &,- 51 & 607 \\
\hline
\end{tabular}

** $p<, 01: * p<.05$

Finalmente, para evaluar la relación entre las variables demográficas y las distintas dimensiones de inclusión -controlando la colinealidad de la edad y el semestre que cursan los alumnos- se estimaron cinco ecuaciones de regresión múltiple utilizando 
como predictores el sexo, la edad y el semestre, y como variable dependiente la práctica educativa inclusiva, el derecho a la inclusión, la inclusión de minorías, la formación docente inclusiva y la exclusión sociocultural. Las variables predictoras se ingresaron a través del método Enter.

En la tabla 9 se muestran los coeficientes de regresión para los predictores incluidos en los modelos finales.

La primera regresión múltiple se utilizó para contrastar la relación entre la práctica educativa inclusiva y el sexo, la edad y el semestre cursado por el estudiante. La ecuación final incluyó como predictores significativos a las variables sexo y semestre, explicando el $12,4 \%$ de la varianza de la práctica inclusiva.

La segunda regresión múltiple se utilizó para contrastar la relación entre el derecho a la inclusión y el sexo, la edad y el semestre cursado por el estudiante. La significación de la ecuación final indica que los predictores incluidos en el modelo no se relacionan con el derecho a la inclusión.

La tercera regresión múltiple se utilizó para contrastar la relación entre la inclusión de minorías y el sexo, la edad y el semestre cursado por el estudiante. La ecuación final incluyó como predictores significativos a las variables sexo y semestre, explicando el 4,9\% de la varianza de la inclusión de minorías.

La cuarta regresión múltiple se utilizó para contrastar la relación entre la formación docente inclusiva y el sexo, la edad y el semestre cursado por el estudiante. La ecuación final incluyó como predictor significativo a la variable semestre, explicando el 2,9\% de la varianza de la formación docente inclusiva.

La quinta regresión múltiple se utilizó para contrastar la relación entre la exclusión sociocultural y el sexo, la edad y el semestre cursado por el estudiante. La significación de la ecuación final indica que los predictores incluidos en el modelo no se relacionan con la exclusión socio cultural.

Tabla 9. Coeficiente de regresión de dimensiones de inclusión en variables socio-demográficas.

\begin{tabular}{|c|c|c|c|c|c|c|c|c|c|c|c|c|c|c|c|}
\hline & \multicolumn{3}{|c|}{$\begin{array}{l}\text { Práctica } \\
\text { educativa } \\
\text { inclusiva }\end{array}$} & \multicolumn{3}{|c|}{$\begin{array}{l}\text { Derecho } \\
\text { a la } \\
\text { inclusión }\end{array}$} & \multicolumn{3}{|c|}{$\begin{array}{l}\text { Inclusión } \\
\text { de } \\
\text { minorías }\end{array}$} & \multicolumn{3}{|c|}{$\begin{array}{l}\text { Formación } \\
\text { docente } \\
\text { inclusiva }\end{array}$} & \multicolumn{3}{|c|}{$\begin{array}{l}\text { Exclusión } \\
\text { socio } \\
\text { cultural }\end{array}$} \\
\hline & b & ES & $\mathbf{R}^{2}$ & b & ES & $\mathbf{R}^{2}$ & b & ES & $\mathbf{R}^{2}$ & b & ES & $\mathbf{R}^{2}$ & b & ES & $\mathbf{R}^{2}$ \\
\hline $\begin{array}{l}\text { Paso } \\
1\end{array}$ & & &, $124 * \star *$ & & & ,015 & & &, $049 * * *$ & & &, $029 * * *$ & & &, 002 \\
\hline Edad & ,013 & ,009 & & ,017 & ,009 & & ,012 & , 010 & &,- 004 & ,010 & & ,005 & ,008 & \\
\hline Sexo &, $250 * * *$ & ,053 & &,- 057 & ,058 & & , $187^{\star \star}$ & ,059 & & ,014 & ,059 & & ,029 & ,051 & \\
\hline Sem. &, $099 * * \star$ & ,023 & & ,006 & ,025 & &, $053^{*}$ & ,025 & &,$- 074 \star \star$ & ,026 & &,- 010 & ,022 & \\
\hline
\end{tabular}

Nota: ES = error estándar, $\mathrm{b}=$ coeficiente de regresión no estandarizado. La ecuación final fue $F(3,359)=17,01 ; p<, 001$

para Práctica educativa inclusiva; $(F(3,359)=1,86 ; p=, 136)$ para Derecho a la inclusión; $F$ $(3,359)=6,15 ; p<, 001$

para Inclusión de minorías; $F(3,359)=3,57 ; p=, 014$ para Formación docente inclusiva y $F$ $(3,359)=, 22 ; p<, 881$ para Exclusión socio cultural.

*** $p<, 001 ; * \star p<, 01 ; * p<, 05$ 


\section{Discusión}

En el año 2015 la UCM actualizó su modelo educativo a fin de reforzar la pertinencia del mismo. Dichos ajustes han quedado plasmados en las políticas institucionales y desde allí se han trasladado a la práctica docente implementándose planes de seguimiento de la puesta en práctica de la innovación curricular en las diferentes carreras. Se han intentado visualizar los elementos facilitadores y obstaculizadores así como la incidencia de la resistencia de la cultura institucional frente a cualquier cambio.

Los cambios asociados a nuevas lógicas de formación universitaria pretenden mejorar la vinculación de la misma con los requerimientos de los mercados laborales, además de empoderar a la comunidad educativa en la gestión de sus derechos y en la optimización de su potencial profesional. Con ello se contribuye a aumentar las posibilidades de convivencia por la vía de la educación integral y humanista. En este sentido la inclusión en los procesos formativos pasa a ser una temática de real importancia en los contextos formativos y socioculturales donde deberán desenvolverse los futuros profesores que esta casa de estudios forma.

No obstante, es necesario destacar que si bien está la intención de armonizar procesos y acciones, los mismos resultan contradictorios con el modelo de desarrollo económico y social capitalista neoliberal circundante, desde donde se evidencia desigualdad y segregación (Lerena \& Trejos, 2015), supuestos que las universidades consciente o inconscientemente reproducen. Por tanto, para que la transformación social sea posible desde los espacios formativos es necesario que los cambios se realicen sistémicamente y que los mecanismos de reproducción se enfrenten con "elementos de oposición, en una dinámica de tensiones desde donde creemos posible generar los reales procesos inclusivos en las instituciones educativas" (Lerena y Trejos, 2015. p. 156).

Por una parte, esta investigación aporta información interesante en tanto permite conocer las actitudes que los estudiantes de pedagogía tienen respecto a la inclusión, sus limitaciones y percepciones. Por otra parte, las evidencias de la validez de constructo aportadas por el AFC permiten contar con instrumentos válidos y fiables que permiten evidenciar las opiniones de los estudiantes.

En esta investigación se obtuvieron evidencias de validez sobre 5 dimensiones de la inclusión: práctica educativa inclusiva, derecho a la inclusión, inclusión de minorías, formación docente inclusiva y exclusión sociocultural, a partir de la exploración de las propiedades psicométricas de la escala de actitudes en una muestra de estudiantes universitarios de diversas carreras de pedagogía en la UCM Talca.

A nivel general se obtuvieron indicadores adecuados, comparables a los obtenidos en otros contextos educativos tanto en secundaria como en educación superior (Colmenero y Pegalajar, 2015; Zambrano, Córdoba y Arboleda, 2012) evidenciándose que las propiedades psicométricas de los ítems son adecuadas (Carretero-Dios y Pérez, 2005; Muñiz, 1992). Esto permite concluir que la escala de actitudes hacia la inclusión presenta características psicométricas aceptables, que muestra, en general, una adecuada consistencia interna y que sus elementos poseen un adecuado poder de discriminación.

A partir del análisis de la estructura interna es posible definir 5 factores que explican la práctica educativa inclusiva, el derecho a la inclusión, la inclusión de minorías, la formación docente inclusiva y la exclusión sociocultural, para los estudiantes de pedagogía de la UCM. Al indagar acerca de las actitudes -entendiéndolas como el sistema por el cual el ser humano determina su relación con el medio ambiente (Alcántara, 1992)-, en cuanto al sexo se constató -a favor de las mujeres- que estas poseen una adecuada actitud hacia la inclusión a diferencia de los varones (Pegalajar y Colmenero, 2017). Si bien esta diferencia pudiera deberse a su mayor representatividad, también podría apuntar a que efectivamente 
las mujeres han logrado desarrollar mayores estrategias inclusivas que los hombres, específicamente en la práctica educativa inclusiva y en inclusión de minorías.

Se podría pensar que a mayor edad los estudiantes tienen mejor actitud hacia la inclusión. Sin embargo, al ingresar las variables semestre y edad se observa que la primera variable se relaciona mejor con la actividad inclusiva. Esto indica que en la medida que los estudiantes avanzan en el proceso formativo mejora su actitud a la inclusión (Mula et al., 2002), lo cual podría deberse a la madurez cognitiva o bien al proceso educativo formal en que se ven inmersos. En este sentido, los hallazgos coinciden con otros estudios locales que sostienen que las actitudes se ven favorecidas cuando los profesores incorporan en su práctica de aula discursos y componentes inclusivos (Sánchez, Díaz, Sanhueza y Friz, 2008) y que la universidad debe brindar los conocimientos y herramientas necesarios para dar respuesta a la diversidad de estudiantes y sus necesidades, dotándoles de competencias para desarrollar prácticas inclusivas que les permitan afrontar los retos educativos. No obstante, la educación inclusiva "no puede ser impuesta: requiere todo el proceso de la participación y convicción de los actores" (Lerena y Trejos, 2015. p. 157).

\section{Conclusiones}

La escala de actitudes hacia la inclusión es fiable, con una dimensionalidad que replica el contenido teórico y unos indicadores de consistencia interna y validez de constructo adecuados. Sin embargo, al ser un instrumento genérico que intenta develar las distintas expresiones de inclusión y/o discriminación presentes en los sistemas escolares formales, no profundiza acerca de las peculiaridades de cada una de ellas ni de las posibles intersecciones y/o superposiciones entre las distintas posibles exclusiones.

Por una parte, y pese a que los resultados parecen indicar una buena bondad psicométrica de la escala, es necesario reseñar que los datos encontrados difícilmente podrían enmarcarse como conclusiones definitivas puesto que, si bien la escala tiene una función importante en la percepción de los participantes, no existen escalas perfectas, o bien nos encontramos con personas que responden pensando en lo socialmente deseable más que en lo que sienten. Por otra parte, si bien se procuró administrar la escala en condiciones óptimas (tiempo, rapport, instrucciones) siempre existe un margen de error que afecta su confiabilidad (Nunnally \& Bernstein, 1994; Reidl-Martínez, 2013).

Asimismo, si bien los análisis realizados permiten contar con un instrumento fiable para abordar el estudio de la inclusión en el contexto universitario chileno, sería de gran relevancia estudiar el comportamiento de la escala en una población más amplia y contrastar los resultados para validar el instrumento.

Finalmente, al ser un instrumento genérico, las conclusiones no permiten profundizar acerca de las especificidades o condiciones en que tiene lugar la exclusión de las distintas comunidades que el estudio intenta visibilizar. 


\section{Referencias bibliográficas}

Alcántara, J. A. (1992). Cómo educar las actitudes. Barcelona, España: CEAC.

Arnáiz, P. (2003). Educación inclusiva: una escuela para todos. Málaga, España: Aljibe.

Avramidis, E., Bayliss, P. \& Burden, R. (2000). A survey into mainstream teachers' attitudes toward the inclusion of children with special educational needs in the ordinary school in one local education authority. Educational Psychology, 20(2), 191-195.

Blanco, R. (2006). La equidad y la inclusión social: uno de los desafíos de la educación y la escuela hoy. Revista electrónica iberoamericana sobre calidad, eficacia y cambios en educación, 4(3), 1-15. Recuperado de http://www.rinace.net/arts/vol4num3/art1.pdf

Blanco, R. (2008). Marco conceptual sobre la educación inclusiva. Conferencia presentada en Centro Internacional de Conferencias, Ginebra. Recuperado de http://www.ibe.unesco. org/fileadmin/user_upload/Policy_Dialogue/48th_ICE/CONFINTED_48_Inf_2_Spanish.pdf

Blanco, R. (2010) (Coord.). El derecho de todos a una educación de calidad. Revista latinoamericana de educación inclusiva, 4(2), 25-153.

Booth, L. (1996). A perspective on inclusion from England. Cambridge Journal of Education, 26(1), 87-99.

Brown, T. (2006). Confirmatory factor analysis for applied research. New York: The Guilford Press.

Calvo, G. (2013). La formación de docentes para la inclusión educativa. Páginas de educación, 6(1), 19-35. Recuperado de http://www.scielo.edu.uy/scielo.php?script=sci arttext\&pid=S1688-74682013000100002\&Ing=es\&tIng=es

Campbell, J., Gilmore, L. \& Cuskelly, M. (2003). Changing student teachers' attitudes towards disability and inclusion. Journal of Intellectual and Developmental Disability, 28 (4), 369-379.

Cardona, M. C. (2002). Introducción a los métodos de investigación en educación. Madrid, España: Editorial EOS.

Cardona, M. C. (2006). Diversidad y educación inclusiva: enfoques metodológicos y estrategias para una enseñanza colaborativa. Madrid, España: Pearson-Prentice Hall.

Carretero-Dios, H. \& Pérez, C. (2005). Normas para el desarrollo y revisión de estudios instrumentales. International Journal of Clinical and Health Psychology, 5 (3), 521-551.

Carretero-Dios, H. \& Pérez, C. (2007). Standards for the development and review of instrumental studies: Considerations about test selection in psychological research. International journal of clinical and health psychology, 7(3), 863-882. Recuperado de http://www.redalyc.org/pdf/337/33770319.pdf 
Colmenero, M. \& Pegalajar, M. del C. (2015). Cuestionario para futuros docentes de educación secundaria acerca de las percepciones sobre atención a la diversidad: construcción y validación del instrumento. Questionnaire for Future Teachers of Secondary Perceptions about Attention to Diversity: Design and Validation of the Instrument. Estudios sobre educación, 29, 165-189. doi: 1015581/004.29.165-189.

Echeita, G. (2013). Inclusión y exclusión educativa. De nuevo voz y quebranto. Revista iberoamericana sobre calidad, eficacia y cambio en educación, 11(2), 99-118.

Frias, D. (2011). Alfa de Cronbach y consistencia interna de los ítems de un instrumento de medida. Recuperado de http://www.uv.es/ friasnav/AlfaCronbach.pdf

Huh, J., Delorme, D. E. \& Reid, L. N. (2006). Perceived third-person effects and consumer attitudes on prevetting and banning DTC advertising. Journal of Consumer Affairs, 40 (1), 90-116. doi:10.1111/j.1745-6606.2006.00047.x

Infante, M. (2010). Desafíos a la formación docente: inclusión educativa. Estudios pedagógicos (Valdivia), 36(1), 287-297.

Jackson, D. L., Gillaspy Jr., J. A. \& Purc-Stephenson, R. (2009). Reporting practices in confirmatory factor analysis: An overview and some recommendations. Psychological Methods, 14, 6-23.

Kline, R. B. (2013). Exploratory and confirmatory factor analysis. En Y. Petscher \& C. Schatsschneider (Eds.), Applied quantitative analysis in the social sciences (pp. 171-207). New York: Routledge.

Lerena, B. \& Trejos, J. (2015). Sobre la posibilidad de una educación inclusiva bajo el actual modelo de desarrollo económico y social chileno. Revista latinoamericana de educación inclusiva RINACE, 9(2), 145-160. Recuperado de http://www.rinace.net/rlei/numeros/vol9num2/art8.pdf

López Melero, M. (1995). Diversidad y cultura: una escuela sin exclusiones. Kikiriki, 38, 26-38. Loreman, T., Forlin, C. \& Sharma, U. (2007). An international comparison of pre-service teacher attitudes towards inclusive education. Disability Studies Quarterly, 27(4). doi: http:// dx.doi.org/10.18061/dsq.v27i4.53.

Mula, A. (dir.). (2002). Incidencia de las actitudes y expectativas de alumnos y profesores sobre el desarrollo del programa de integración. Universidad de Alicante.

Muñiz, J. (1992). Medio siglo de teoría de respuesta a los ítems. Anuario Psicología, 52, 41-66.

Muñoz, M. L., López, M. \& Assaél, J. (2015). Concepciones docentes para responder a la diversidad: ¿Barreras o recursos para la inclusión educativa? Psicoperspectivas. Individuo y sociedad, 14(3), 68-79. doi: 10.5027/psicoperspectivas-vol14-issue3-fulltext-646

Nunnally, J. C. \& Bernstein, I. H. (1994). Psychometric Theory. New York: McGraw-Hill Inc.

Pegalajar, M. del C. \& Colmenero, M. de J. (2017). Actitudes y formación docente hacia la inclusión en educación secundaria obligatoria. Revista electrónica de investigación educativa, 


\section{9, 84-97. doi.org/10.24320/redie.2017.19.1.765}

Pozo, J., Scheuer, N., Pérez, M., Mateos, M., Martín, E. \& De la Cruz, M. (2006). Nuevas formas de pensar la enseñanza y aprendizaje: las concepciones de profesores y alumnos. Barcelona: Graó.

Programa de las Naciones Unidas para el Desarrollo (2000). Informe sobre desarrollo humano 2000. Madrid: Mundi Prensa.

Reidl-Martínez, L. M. (2013). Metodología de investigación en educación médica. Confiabilidad en la medición. Investigación en educación médica, 2(6), 107-111.

Sales Ciges, A., Moliner García, O. \& Sanchiz Ruiz, M. L. (2001). Maestros y maestras críticos. Cuadernos de pedagogía, 307, 39-42.

Sánchez, A., Díaz, C., Sanhueza, S. \& Friz, M. (2008). Percepciones y actitudes de los estudiantes de pedagogía hacia la inclusión educativa. Estudios pedagógicos, 34(2), 169-178. Recuperado de http://www.scielo.cl/scielo.php?script=sci_arttext\&pid=S0718-07052008000200010

Sanhueza, S., Granada, M. \& Bravo, L. (2012). Actitudes del profesorado de Chile y Costa Rica hacia la inclusión educativa. Cuadernos de pesquisa, 42(147), 884-899.

Sanya, E. O., Salami, T. A. T., Goodman, O. O., Buhari, O. I. N. \& Araoye, M. O. (2005). Perception and attitude to epilepsy among teachers in primary, secondary and tertiary educational institutions in middle belt Nigeria. Tropical Doctor, 35 (3), 153-156. doi/10.1258/0049475054620905

Tait, K. \& Purdie, N. (2000). Attitudes toward disability: teacher education for inclusive environments in an Australian university. International Journal of Disability, Development and Education, 47(1), 25-38.

Vezub, L. (2007). La formación y el desarrollo profesional docente frente a los nuevos desafíos de la escolaridad. Revista de curriculum y formación del profesorado, 11, 2-33. Recuperado de https://www.ugr.es/ recfpro/rev111ART2.pdf

Zambrano, R., Córdoba, V. \& Arboleda, C. (2012). Percepciones de la inclusión escolar en estudiantes de educación secundaria. Pensando psicología, 8(15), 18-29.g

${ }^{1}$ Investigación realizada en el marco del Proyecto PMI Formación Inicial de Profesores UCM 1310. 